\title{
Architecture of the Digital Twin in Product Validation for the Application in Virtual-Physical Testing to Investigate System Reliability
}

\author{
Felix Leitenberger ${ }^{1}$, Thomas Gwosch ${ }^{1}$, Sven Matthiesen ${ }^{1 *}$ \\ ${ }^{1}$ IPEK - Institute of Product Engineering at the Karlsruhe Institute of Technology (KIT) \\ * Corresponding author: \\ Univ.-Prof. Dr-Ing. Sven Matthiesen \\ IPEK - Institute of Product Engineering \\ Kaiserstr. 10 (Building 10.23) \\ 76131 Karlsruhe \\ Phone: +49 721 608-47156 \\ E-Mail: sven.matthiesen@kit.edu
}

\begin{abstract}
The digital twin offers new possibilities for product validation in the application of virtual-physical testing. A more concrete description of the digital twin in product validation is missing and therefore will be specified here. For this aim, a single-case study was conducted using an electrohydrostatic actuator. This system is influenced by many interactions due to its integral design and is thus representative of a complex system. The characterization of the digital twin in product validation is done by specifying the model quality, interoperability and integration levels. The research results of the digital twin are used and linked to the state of research from product validation. An architecture represents these characteristics and thus allows transferability to other systems.
\end{abstract}

\section{Keywords}

Digital Twin, Product Validation, Testing, System Reliability, Electrohydrostatic Actuator 


\section{Motivation and state of research}

Early testing of product properties is an important part of product validation in terms of frontloading to avoid iterations on physical prototypes [1]. In this context, the proof of system reliability with regard to functional fulfillment is often associated with a high level of effort, which can be provided by physical or virtual testing. Physical testing requires expertise in the design of test benches. Virtual testing requires a high level of system knowledge for the modeling of the system. This is not always equally available for all subsystems, making mixed virtualphysical testing useful.

For parallel virtual-physical testing in product validation, the concept of the digital twin can be used. There is no precise definition of the digital twin, as the term is still in a state of transformation. Depending on the field of research, however, more detailed and specified definitions are available, which have been collected by Stark and Damerau, Jones or Tao, among others [2-4]. In this paper, the definition according to Stark will be followed:

"A Digital Twin is the digital representation of a unique asset (product, machine, ...), that compromises its properties, condition and behavior by means of models, information and data". [5] Figure 1 shows this relationship, which originates from Grieves.

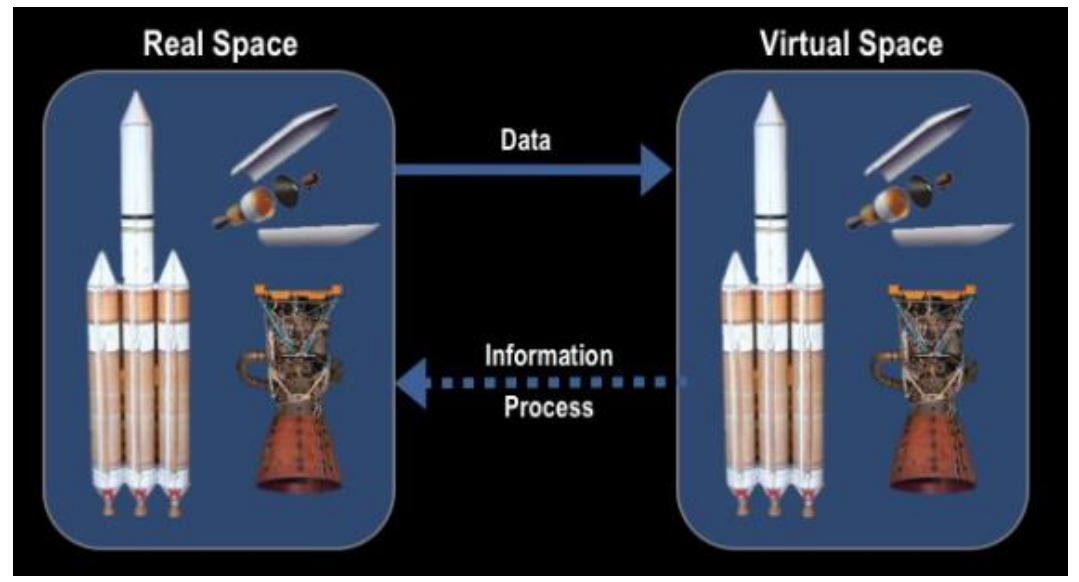

Figure 1: Concept of the digital twin by Grieves [6]

The digital twin in product validation offers new possibilities for generating knowledge and validating product properties and thus supports frontloading [7]. In addition, the further utilization of the generated data in subsequent phases results in new application opportunities, which in turn can be applied in product development [8]. The digital twin is currently increasingly investigated and applied in production engineering and the subsequent phases of the product life cycle [9]. A transfer of the abstract descriptions of the digital twin to product development, especially to product validation, has not yet taken place sufficiently [10]. Therefore, a more concrete description is lacking for the application of digital twins in product validation $[11,12]$.

Digital twins are classified concerning their application focus by characterizing model quality, interoperability and integration levels [8].

In terms of the integration levels, there is a precise description by Kritzinger in production engineering. The digital twin only exists if there is a fully automatic data exchange between the physical and virtual world. A Digital Shadow is when there is an automatic exchange of data from the physical to the virtual world. However, data is then exchanged manually from the virtual to the physical world. The smallest level is the digital model, where data exchange must be executed manually. [13] 
The research aim is to concretize the digital twin in product validation based on the characteristics concerning model quality, interoperability and integration levels. An architecture for the digital twin in product validation is presented. The application focus is described.

\section{Materials and methods}

\subsection{Single case study}

To concretize the digital twin in product validation and to create a suitable architecture, a single case study is conducted. An electrohydrostatic actuator (EHA) from aviation is used as the system to be analyzed and is suitable as a reference system due to its multiphysical dimensionality. The integral design of the EHA poses new challenges for product validation due to the high demands on reliability and robustness in aviation. The interactions between the components have a strong impact on the function of the EHA, which requires a high level of system knowledge to create a sufficient virtual model.

Using the EHA as an example, the characteristics of the digital twin concerning model quality, interoperability and integration levels for the application in product validation are specified for this purpose. To do so, the abstract characterization of the digital twin, which is known from the state of research, is used and applied to product validation by studying the EHA. To aim this, the state of research from the fields of modeling and simulation, as well as virtual-physical testing, is used and combined with the findings of the digital twins from other disciplines.

\subsection{Electrohydrostatic Actuator}

In this context, the EHA represents a prototype whose functions are to be validated and optimized. The EHA consist of the components electric motor, hydraulic pump, hydraulic system and hydraulic cylinder, shown in Figure 2. The hydraulic system includes the piping system between the hydraulic pump and cylinder. There is no valve control in this EHA. The volume flow is controlled directly via the speed of the pump.

The integral construction of these components creates interactions that would not occur individually. However, the combination of the given components poses new challenges for developers, as these interactions require a high level of system knowledge.

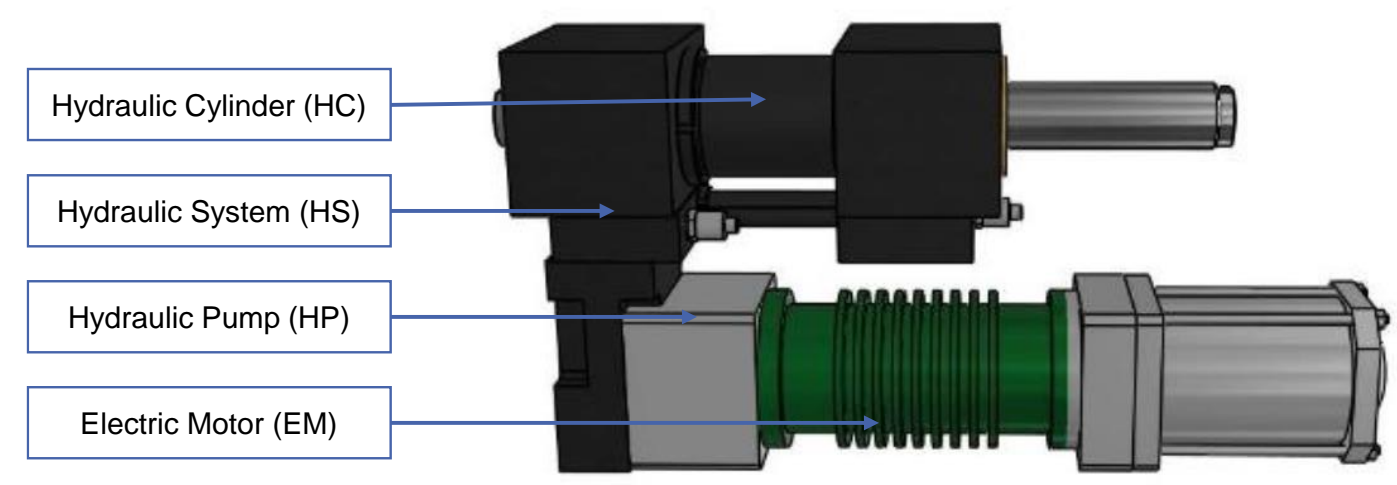

Figure 2: Design of the electrohydrostatic actuator (EHA) to be investigated

For testing on the test bench, an inverter is necessary to provide the required power for the synchronous electric motor. The environment is described by an axial load, which can be applied by a spring package or another linear actuator. The focus is on the testing of the dynamic system behavior. 


\section{Results}

The results of the single-case study are described in the following, step by step. First, the application focus is concretized. Then, the characteristics of the digital twin, concerning model quality, interoperability and integration levels, for the application in product validation are discussed in detail. Finally, the architecture of the digital twin in product validation is described.

\subsection{Application Focus}

The digital twin is to be described for the application in product validation. In product validation, the physical world is limited to physical prototypes and test benches. The prototypes represent the system to be tested and are also called unit-under-test (UUT), system-underinvestigation (Sul) or system-in-development (SiD). The test bench represents the environment and other boundary conditions. In the context of product validation, the virtual world can be represented with a suitable simulation. The objective is to obtain a digital representation with which the function to be investigated can be tested for its performance and system reliability. Both the test bench and the prototype can exist virtually and physically. Due to the coupling between the physical and virtual worlds, two aspects of the application are basically in the focus: Knowledge can be generated through fast, low-cost and low-risk iterations in the simulation. An exchange of data between the physical and virtual worlds, also called twinning, additionally allows new insights to be gained. The physical world continues to be used for validating the function and system reliability. The following figure shows the basic understanding.

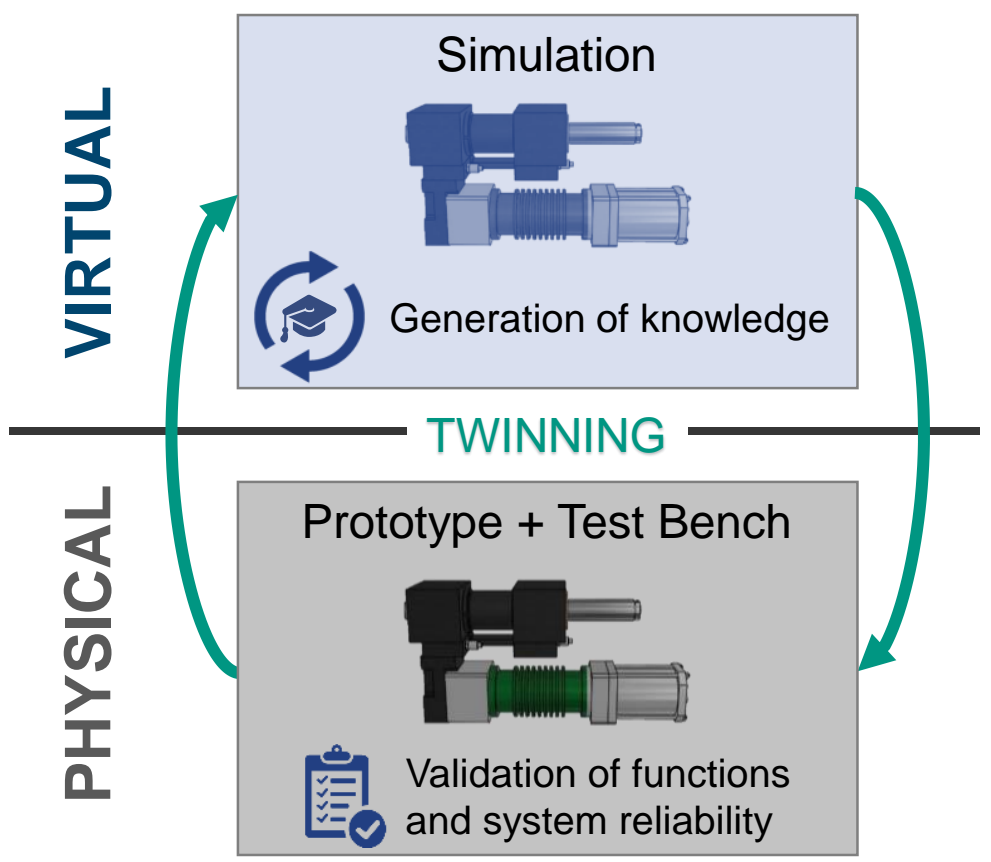

Figure 3: Basic understanding of the digital twin in product validation to describe the application focus adapted from [7]

\subsection{Model Quality}

The model quality defines how realistically a digital twin represents its physical product instance [8]. In this context, the scope and accuracy of the simulation model have to be specified. 


\subsubsection{Scope}

For the development of the EHA, the primary function fulfillment and system reliability are in the foreground. The function to be investigated is the system behavior at the interface to the environment.

The created simulation model must therefore represent the dynamic behavior. Since the variables are only functions of time and not dependent on the spatial coordinates, modeling with lumped parameters is sufficient for this application. The simulation is therefore based on multiphysics modeling, in which the relevant domains are considered to describe the dynamic system behavior. Suitable software is MATLAB ${ }^{\circledR}$ Simulink ${ }^{\circledR}$, which was used here. In the case of the EHA, the mechanical, electrical and hydraulic domains were identified by considering the main power flow. According to the boundary conditions during flight, it is additionally reasonable to consider the thermal domain. System-describing parameters such as the electric resistance of the copper windings in the electric motor are temperature-dependent.

In the first instance, the subsystems must be defined based on their function. This can be realized by using the description of the process elements transformer, converter, source and sink. The relevant domains can then be assigned to each of these elements. Thus, the power electronics represents a transformer of electrical energy. The electric motor is a converter of electrical into mechanical energy. The hydraulic pump is a converter from mechanical to hydraulic energy and the hydraulic cylinder from hydraulic to mechanical energy. The hydraulic system is a transformer of hydraulic energy. Figure 4 shows the assignment of the relevant domains to the respective subsystems. This describes the scope of the simulation.

One requirement of the simulation is the transfer of the main power flow between the individual subsystems and the associated relevant interactions. The interactions must be transferred in order to investigate the system reliability.

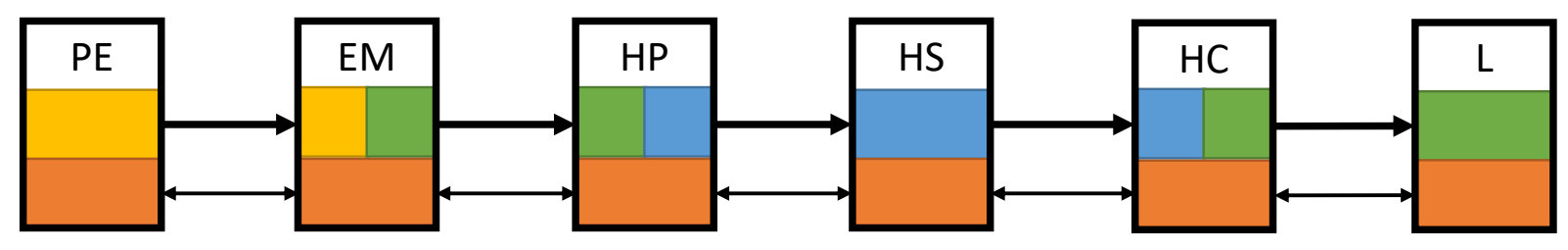

\begin{tabular}{|c|c|c|c|c|}
\hline PE & Power Electronics & $\mathrm{HS}$ & Hydraulic System & Main power flo \\
\hline EM & Electric Motor & $\mathrm{HC}$ & Hydraulic Cylinder & \\
\hline HP & Hydraulic Pump & $\mathrm{L}$ & Load & Inter \\
\hline
\end{tabular}

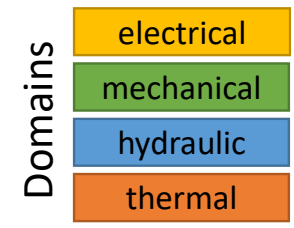

Figure 4: Assignment of the relevant domains to the respective subsystems

Detailed modeling then takes place within these subsystems and is thus closely linked to accuracy.

\subsubsection{Accuracy}

The level of detail within a subsystem can be different. Depending on the knowledge of the system, the modeling within the individual subsystem can thus also be created as a black-box model or white-box model. In white-box modeling, purely analytically derived relations are described via differential equations. In black-box modeling, the model can be created experimentally or can include parts of it. Datasheets can be used for this purpose, for example.

The required accuracy of the simulation can be adapted to the accuracy of the measurement method on the test bench. It does not appear reasonable to demand a higher accuracy from 
the simulation model than the accuracy that can be measured. Absolute and relative deviations of the measured parameters can be used as evaluation criteria.

The interactions between the domains within the individual subsystem must be able to be taken into account, otherwise, no connection between the domains is established. The connections between the domains of the main power flow result from their functional principle. The connections to the thermal domain can be established by considering the losses of the main power flow. Depending on the type, these primarily turn into heat and are therefore further considered in the thermal domain of the respective subsystem.

\subsection{Interoperability}

Interoperability describes the characteristic that the individual digital twins are compatible and can interact with each other. [8]

Due to the structure described, each subsystem can be regarded as an independent and individual digital twin. The interoperability between these subsystems is given by the description of the interfaces using power variables. Power is transferred between the process elements, which can be described as the product of an across-variable and a through-variable, regardless of their domain. Figure 5 shows the interfaces with the power variables between the subsystems. The direction of the arrows describes the direction of the information flow in the simulation.

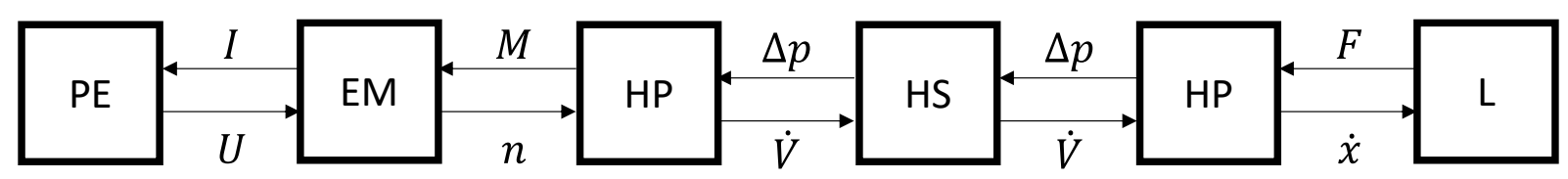

Figure 5: Interfaces between the individual subsystems for interchangeability to ensure interoperability

The interaction of the individual subsystems in the virtual world can be achieved directly by closed modeling. All subsystems are created in the same simulation environment and executed as a complete system. Additional steps, as required for co-simulation, are not necessary.

In the physical world, research knowledge gained from the development of test benches can be used. X-in-the-loop (XiL) test benches are suitable for connecting physical and virtual subsystems using coupling systems, so that testing in the overall system is made possible [14]. XiL test benches also often work with power variables and therefore use the same interface as the simulation model.

The interoperability of the digital twin in product validation is ensured by exchanging power variables between the subsystems, thus enabling the substitution of the subsystems. The interactions between the individual subsystems can be transferred.

\subsection{Integration Level}

The integration level describes the level of data connection between the physical and virtual worlds. [13]

The description of the integration level is thus closely linked to the coupling systems from the XiL test benches. These are used for virtual-physical testing and have a combination of sensor-actuator technology. Since they also work with power variables, they are suitable for the bidirectional data exchange between the physical and virtual worlds.

Coupling systems enable the transfer of interactions between physical and virtual subsystems [15]. This means that automated, bidirectional data exchange can be established, thus fulfilling the requirements for the digital twin. 
The challenge is to develop suitable coupling systems for the relevant domains. In the mechanical domain for rotational movements, there is already a state of the art that has been researched for many years and is widely used $[15,16]$. In contrast, coupling systems for the thermal domain are currently the subject of research [17].

If no actuator is required, a sensor system can also be used instead of a coupling system. This is the case, for example, if the physical subsystems are not spatially separated. Suitable sensors are available for all power variables used here.

Coupling systems used on XiL test benches are ideal for bidirectional data exchange, which is necessary for digital twins to achieve the required level of integration.

\subsection{Architecture}

The architecture represents the combination of the described results and is shown in figure 6. The digital twin with the application context for product validation is described. The characteristics of model quality, interoperability and integration level are discussed and specified. This is done on the system of the EHA but serves to be transferred to other systems as well.

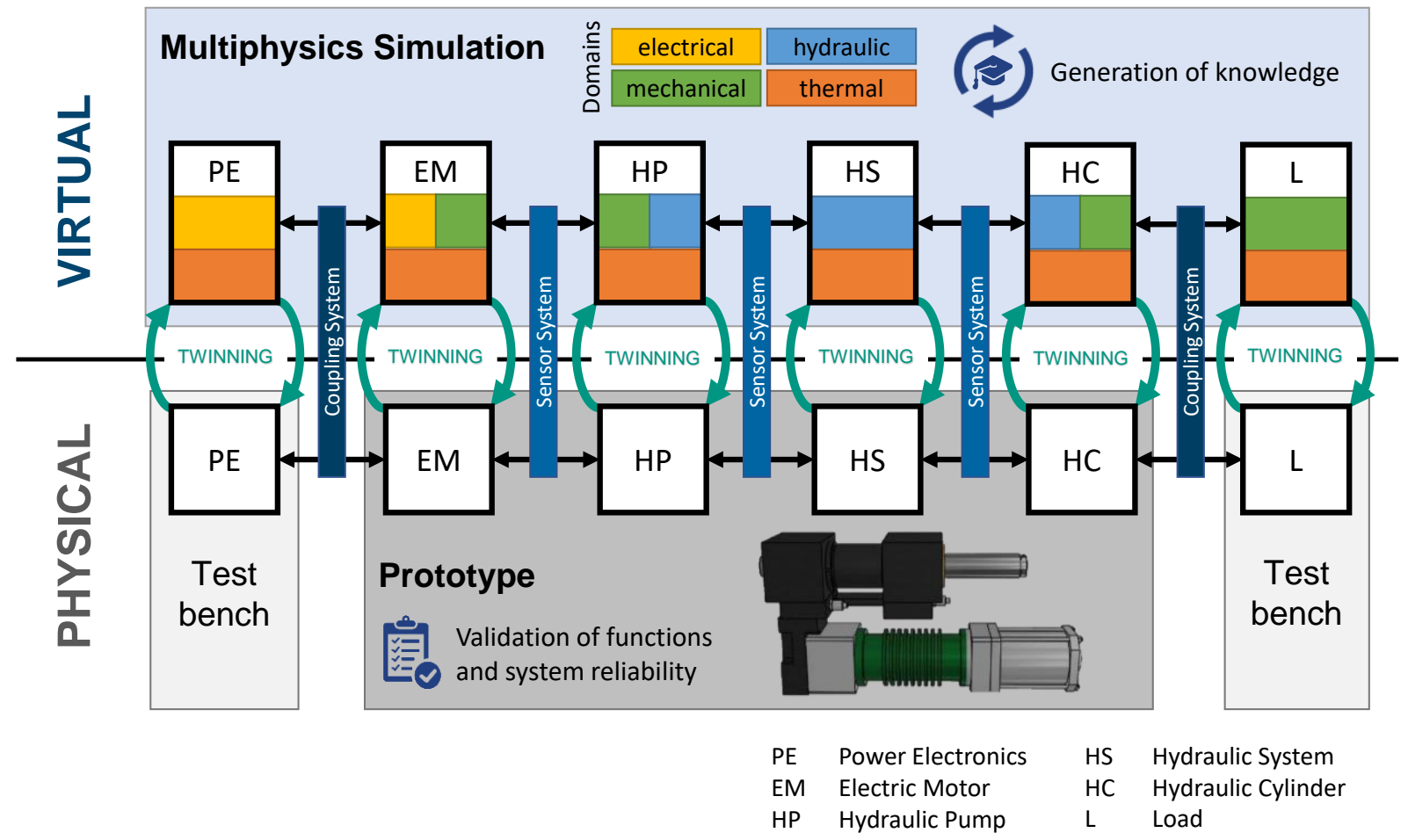

Figure 6: Architecture of the digital twin in product validation for application in virtual-physical testing to investigate system reliability using the example of an EHA

\section{Discussion}

The digital twin was concretized for product validation based on the characteristics concerning model quality, interoperability and integration level and put into context by describing an architecture. However, this is also associated with some limitations. These limitations result primarily from the fact that the description of the digital twin for product validation was investigated from a single case study.

The EHA has a clearly defined power flow that is routed through the various subsystems. An extensive system with several power and information flows, some of which run in parallel 
and thus represent a network, has not yet been created with this description. Logical states of the system have not yet been investigated as in other modeling approaches [18].

The level of detail regarding the model quality is not tightly defined. It has not yet been investigated how the model quality of the individual subsystem affects the behavior of the overall system. Compatibility is given by the interfaces, but it is not clear how the digital twins of different model qualities behave with each other.

Twinning requires coupling or sensor systems that establish bidirectional data exchange between the physical and virtual worlds. There are already coupling systems that can be used for this application. The use of coupling systems is different from previous research in that it considers multiple domains. For the application at the EHA, a coupling system for the thermal domain is required and has therefore to be investigated.

With the coupling systems, automated data exchange between the physical and virtual worlds is technically possible. However, this places high demands on the measurement and control system to be used on the test bench. In this application, the simulation and the test bench must run in parallel. The test bench must not only acquire the data but also perform the computing operations for the simulation in parallel. Changes that are to be applied to the test bench by the simulation are also transferred directly.

\section{Conclusion and future work}

The digital twin is described for the application in product validation using the example of an electrohydrostatic actuator (EHA). The digital twin in product validation is characterized concerning model quality, interoperability and integration level.

The physical world is limited to physical prototypes and test benches. The virtual world is represented by a simulation. This simulation is based on multiphysics modeling and considers all relevant domains. The overall system is divided into subsystems based on its function as a transformer, converter, source or sink. The consideration of the relevant interactions is a requirement for the model quality.

The interoperability between the different subsystems, which can be seen here as independent digital twins, is ensured by using the power variables as a defined interface. This ensures that the individual subsystems are interchangeable.

The integration level can be reached on its highest level using coupling systems of the $\mathrm{X}$-in-the-Loop (XiL) test benches. They ensure fully automatic data exchange between the physical and virtual world and allows to use the term of digital twin.

An architecture is presented, which can visualize the characterization of the digital twin in product validation. This can be used to transfer the digital twin to other systems for the application in virtual-physical testing.

The findings obtained here serve as a starting point for further investigations. The consideration of the thermal domain is to be investigated. For this purpose, coupling systems are to be used, which allow the consideration of thermal interactions. In the future, it should be possible to identify the relevant domains in an early stage of product development.

Based on the described architecture of the digital twin, new validation methods can be developed in the context of virtual-physical testing. The focus is placed on twinning and the use of coupling systems for bidirectional data exchange. This will be tested on different application scenarios on the EHA. The focus is on supporting the parameterization of the simulation, the detection of anomalies on the test bench, the optimization of the control and the increase of efficiency. Many different application scenarios concerning system reliability can be considered and are to be described methodically in such a way that they can be transferred to other systems. 


\section{Acknowledgments}

The project upon which this publication is based was funded by Federal Ministry for Economic Affairs and Energy under the funding number 20Y1910E. The authors of this publication are responsible for its contents.

\section{Literature}

[1] Thomke, Stefan; Fujimoto, Takahiro: The Effect of "Front-Loading" Problem-Solving on Product Development Performance. In: Journal of Product Innovation Management, Vol. 2, 2000, pp. 128-142.

[2] Stark, Rainer; Damerau, Thomas: Digital Twin. In: Chatti, S. und Tolio, T. (Hrsg.): CIRP Encyclopedia of Production Engineering. Springer Berlin Heidelberg, 2019, 66. Jg., pp. 1-8.

[3] Jones, David, et al.: Characterising the Digital Twin: A systematic literature review. In: CIRP Journal of Manufacturing Science and Technology, Vol. 29, 2020, pp. 36-52.

[4] Tao, Fei; Zhang, Meng; Nee, Andrew Yeh Chris: Digital twin driven smart manufacturing. Academic Press, 2019, pp. 7-10.

[5] Stark, Rainer; Kind, Simon; Neumeyer, Sebastian: Innovations in digital modelling for next generation manufacturing system design. In: CIRP Annals, 2017, Vol. 66, No. 1, pp. 169-172.

[6] Grieves, Michael: Digital Twin: Manufacturing Excellence through Virtual Factory Replication. Whitepaper, 2014, https://www.researchgate.net/publication/275211047_Digital_Twin_Manufacturing_Excellence_through _Virtual_Factory_Replication.

[7] Matthiesen, Sven et al.: Systemzuverlässigkeitssteigerung durch effizientes Testen mit KI und Digitalem Zwilling. In: Newsletter Wissenschaftliche Gesellschaft für Produktentwicklung WiGeP, Vol. 1, 2021, p. 11, http://www.wigep.de/fileadmin/download/wigep/WiGeP_News_1_2021.pdf

[8] Stark, Rainer et al.: WiGeP-Positionspapier: „Digitaler Zwilling“. In: ZWF Zeitschrift für wirtschaftlichen Fabrikbetrieb, 2020, pp. 47-50.

[9] Tao, Fei. et al.: "Digital twin-driven product design framework", International Journal of Production Research, Vol. 57 No. 12, 2019, pp. 3935-3953.

[10] Trauer, Jakob et al.: What is a Digital Twin? - Definitions and Insights from an Industrial Case Study in Technical Product Development. In: Proceedings of the Design Society: DESIGN Conference. Cambridge University Press, 2020, pp. 757-766.

[11] Sturm, Carolin et al.: Creation of Digital Twins - Key Characteristics of Physical to Virtual Twinning in Mechatronic Product Development. In: ICED 23 ${ }^{\text {rd }}$ International Conference on Engineering Design, 2021 (in publication)

[12] Schleich, Benjamin et al.: Shaping the digital twin for design and production engineering. In: CIRP Annals, 2017, Vol. 66, No. 1, pp. 141-144.

[13] Kritzinger, Werner et al.: Digital Twin in manufacturing: A categorical literature review and classification. In: IFAC-PapersOnLine, 2018, Vol. 51, No. 11, pp. 1016-1022.

[14] Matthiesen, Sven et al.: X-in-the-Loop in der Gerätebranche: Ein ganzheitliches Validierungsframework unter Berücksichtigung der Wechselwirkungen zwischen Anwender, Gerät und Anwendung. In: Stuttgarter Symposium für Produktentwicklung (SSP), Fraunhofer IOA, Germany, 2013, pp. 1-10.

[15] Albers, Albert et al.: Koppelsystems: Obligatory Elements within Validation Setups. In: Proceedings of DESIGN 2016, 2016, pp. 109-118.

[16] Gwosch, Thomas; Steck, Michael; Matthiesen, Sven: Virtual Coupling of Powertrain Components: New Applications in Testing. In: ASIM - Simulation technischer Systeme, Grundlagen und Methoden in Modellbildung und Simulation, 2019, pp. 163-168.

[17] Leitenberger, Felix et al.: Investigations to Consider Thermal Interactions Between Spatially Separated Subsystems: Concept of a Thermal Coupling System for X-in-the-Loop Test Benches. In: Proceedings of the ASME 2021 International Mechanical Engineering Congress and Exposition IMECE2021, 2021 (in publication)

[18] Matthiesen, Sven; Ruckpaul, Anne: New insights on the Contact\&Channel-Approach - modelling of systems with several logical states. In: Marjanović (Hrsg.): DS 70: Proceedings of DESIGN 2012, the 12th International Design Conference, 2012, pp. 1019-1028. 